\title{
Measuring Methods for Lining Erosion of the Blast Furnace*
}

\author{
By Yoshio KAWATE,** Hidekazu SONOI,*** Kanji YOKOE,**** \\ Sei TAKANO***** and Kouji SHIMOMURA ${ }^{* * * * * *}$
}

\begin{abstract}
Synopsis
A new fine multiple thermocouple sensor (FMT sensor) for the measurement of refractory lining erosion of a blast furnace is presented. In the newly developed sensor, the temperature sensing parts of several sheathed thermocouples are arranged longitudinally parallel. They are accommodated in an external sheathing tube in such a manner that these thermocouples are prevented from coming into mutual contact by means of insulating refractory materials. The sensor can simultaneously measure the temperature variations at several positions in the refractory lining.

$A$ method of analyzing the FMT signals (The Trigger Response Method) has also been developed for calculating the remaining thickness of refractory lining in the shaft of a blast furnace by analyzing the timedelay occurring in the propagation of temperature variations in the lining. By applying this method to 19 months of field testing in the shaft of No. 1 Blast Furnace of Kobe Works, it was possible to estimate the extent of erosion of the refractory lining within an error tolerance of less than $5 \%$.

$A$ method of analyzing the temperature distribution in the lining (The Profile Coefficient Method) has also been developed for estimating the profile of lining erosion in the hearth by introducing the Profile Coefficient defined by the solution of the finite element method in which the boundary conditions are determined indirectly by using measured temperatures in the lining. This method was applied in the No. 1 Blast Furnace of Kakogawa Works and its practicability was confirmed when its estimation corresponded to those taken by the dissection studies.
\end{abstract}

\section{Introduction}

It has become very important in recent years to extend the lifetime of the blast furnace. As the blast furnace has increased in size and pressure, the refractory lining has become subject to increasingly rigorous use. If the lining erosion can be measured continuously during the operation of a blast furnace, the measured result becomes very useful for extending the lifetime of the blast furnace. Therefore, a number of studies towards measuring methods for lining erosion have been done, ${ }^{1)}$ but a reliable on-line method which can monitor lining erosion at any time has not yet been perfected.

In order to solve those problems, we have developed a new fine multiple thermocouple sensor (FMT sensor) for measuring the variations in the lining and two analyzing methods for calculating the remaining lining thickness. One of these methods, the Trigger Response Method, can analyze the lining erosion of the shaft. The other method, the Profile Coefficient Method, can analyze the profile of the lining erosion of the hearth.
These techniques were applied to blast furnaces in Kobe Steel Ltd. The outline is described in the following.

\section{Measurements of the Lining Erosion of the Shaft}

Conventionally, the thickness of the remaining refractory lining of a blast furnace is estimated by the temperature distribution through the lining by means of a thermocouple, a heat flow meter, ${ }^{2,3)}$ an infrared ray camera, ${ }^{4)}$ etc. These estimation, however, suffer because the physical properties of the refractory materials are sequentially changed during the operation of a blast furnace and the inner surface temperature can not be determined a priori.

These problems could be solved by using the newly developed fine multiple thermocouple sensor (the FMT sensor) that produces signals which can be analyzed so as to obtain a refractory lining thickness.

The Trigger Response Method of analyzing the signals, which is based on the transmission delay of temperature variation in the refractory lining, has also been developed.

This method was applied to 19 months of field testing in the shaft of No. 1 Blast Furnace of Kobe Works. Its practicability was confirmed when its measurements corresponded to those taken by boring through the lining.

The details of the sensor, the analyzing method and results of the field test are described in the following.

\section{The Structure and Specification of the FMT Sensor}

The fine multiple thermocouple sensor (The FMT sensor) can simultaneously measure temperature variations at a number of positions across the thickness of the lining. The entire FMT sensor assembly is shown in Photo. 1 and a cut away drawing of the FMT sensor is shown in Fig. 1. The temperature sensing points of several sheathed thermocouples are arranged in parallel longitudinally. These thermocouples are accomodated in a second outer sheathed tube and are prevented from coming into mutual contact by means of insulating refractory material. Furthermore, in cross section, the sensor is uniformly configurated with a dummy element connected to the end of each

\footnotetext{
* Based on the paper presented to the symposium of the 102nd ISIJ Meeting, November 1981, at Kyoto-fu Chusho Kigyo Kaikan, Kyoto, (published in Tetsu-to-Hagané, 67 (1981), A129, in Japanese). English manuscript received April 28, 1982. (C) 1982 ISIJ

** $\quad$ Asada Research Laboratory, Kobe Steel Ltd., Gomo-aza-maruyama, Nada-ku, Kobe 657.

*** Mechanical Engineering Research Laboratory, Kobe Steel Ltd., Wakinohama-cho, Chuo-ku, Kobe 651.

**** $\quad$ R \& D Planning Department, Kobe Steel Ltd., Wakinohama-cho, Chuo-ku, Kobe 651.

***** Kobe Works, Kobe Steel Ltd., Nadahama-higashimachi, Nada-ku, Kobe 657.

****** Kakogawa Works, Kobe Steel Ltd., Kanazawamachi, Kakogawa 675-01.
} 
thermocouple. Photograph 2 shows an example of a cross section of the FMT sensor. Any cross section of the FMT sensor is designed to have a geometrically uniform configuration so that the heat flow is kept uniform throughout the length of the sensor.

A reliability test of the FMT sensor was carried out at the slag runner (the slag trough) of the No. 1 Blast Furnace of Kakogawa Works. It turned out that the tip eroded portion of the FMT sensor had coincided with the front surface of the remaining lining but still continued its performance through the remaining part of the sensor.

The specifications of the FMT sensor are summarized in Table 1. The following can be presented

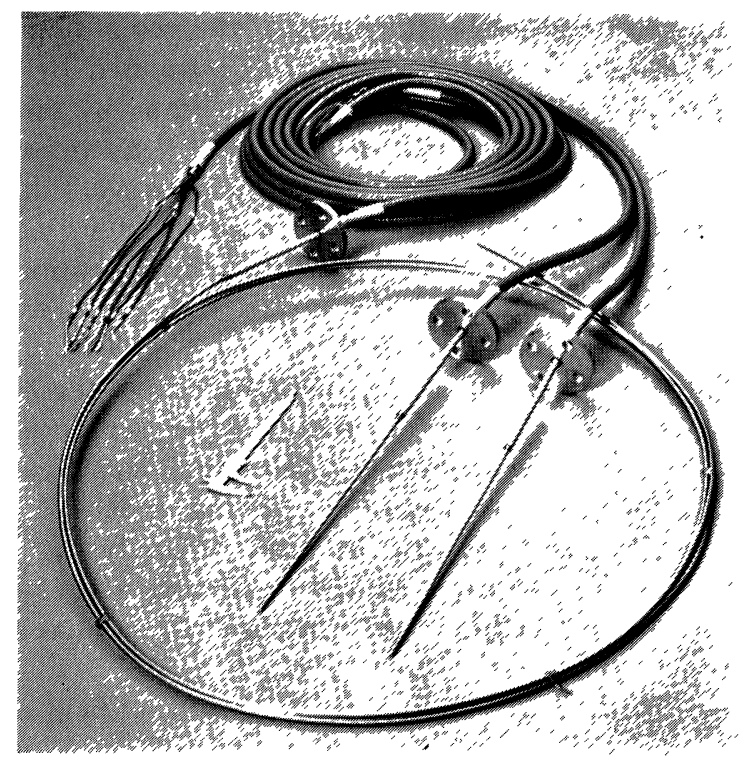

Photo. 1. The FMT sensor assembly.

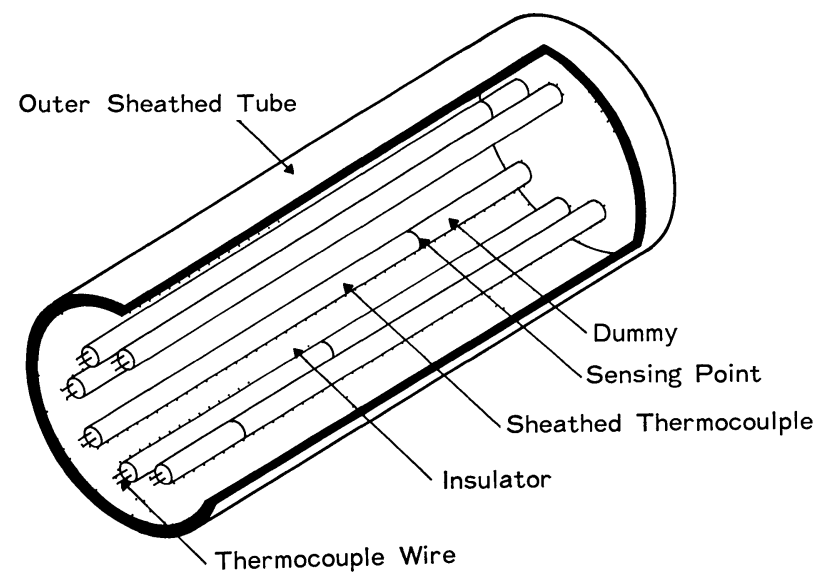

Fig. 1. The FMT sensor construction.

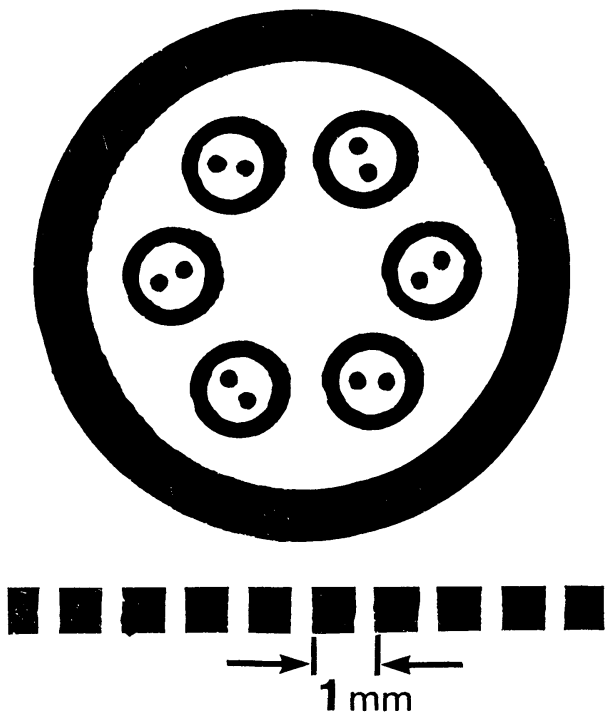

Photo. 2. Cross section of the FMT sensor.

Table 1. Summary of the FMT sensor specification.

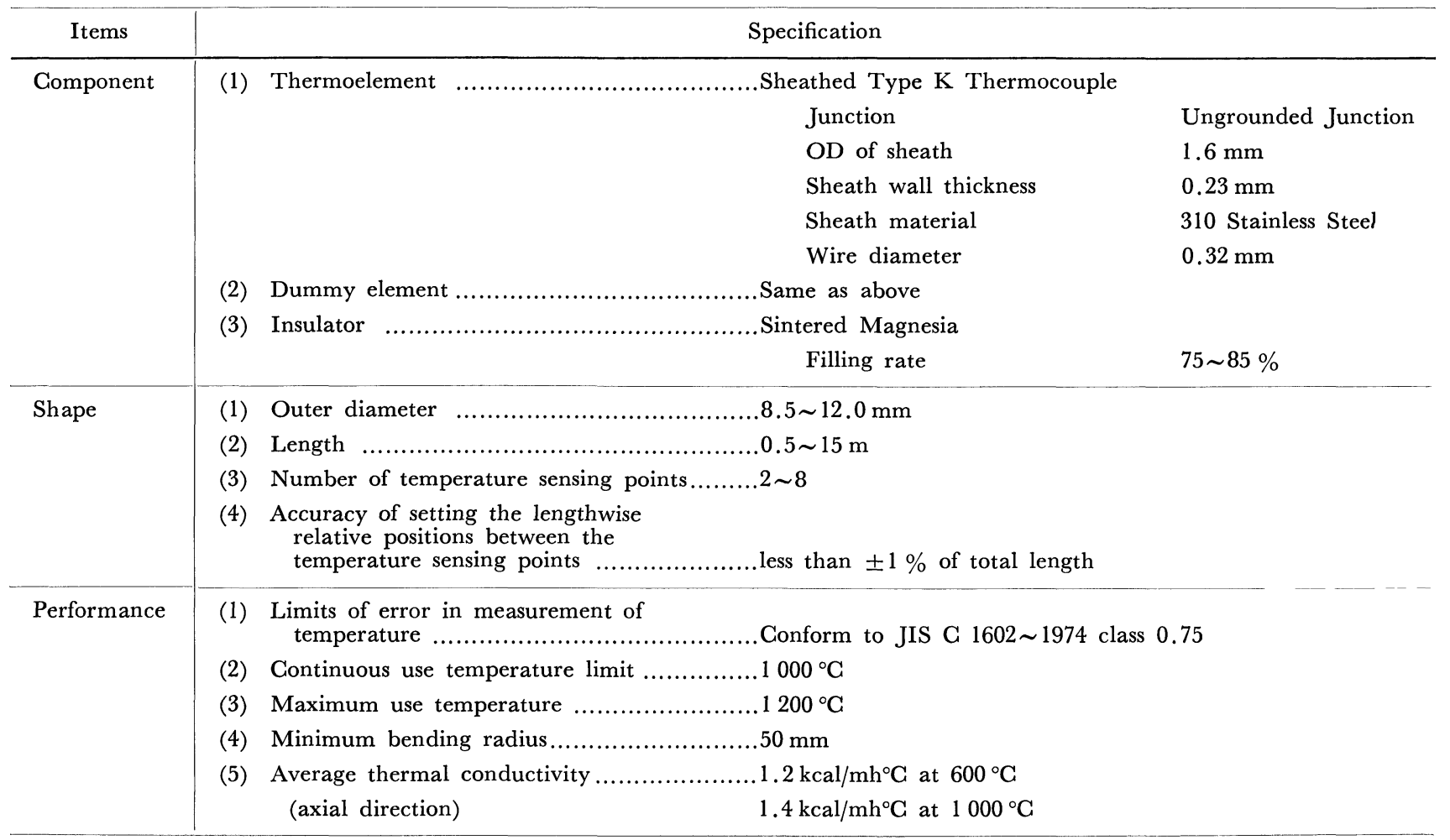


as the characteristics of the FMT sensor contrasted to a conventional sheathed thermometer.

(1) The FMT sensor can measure temperature variations simultaneously at a number of positions across the lining, resulting in a reduction of the number of holes required for thermometers.

(2) The relative longitudinal positions of the temperature-sensing points can be designed before installation.

(3) The mean thermal conductivity of the FMT sensor is nearly equal to the brick lining; therefore, there was hardly any affect on the thermal profile.

(4) The double-sheath structure ensures high resistance to the atmosphere.

(5) The filling rate of the insulating refractory is high and the amount of residual gas is small; therefore, the durability of the sensor can be assured.

\section{Principles of Analysis by the Trigger Response Method}

When the signal "Trigger signal", $T_{t}$, representing the heat variation in a furnace is transmitted into the lining, the variation of temperature called the "Response signal ", $\mathcal{T}_{r i}$, is accordingly transferred at

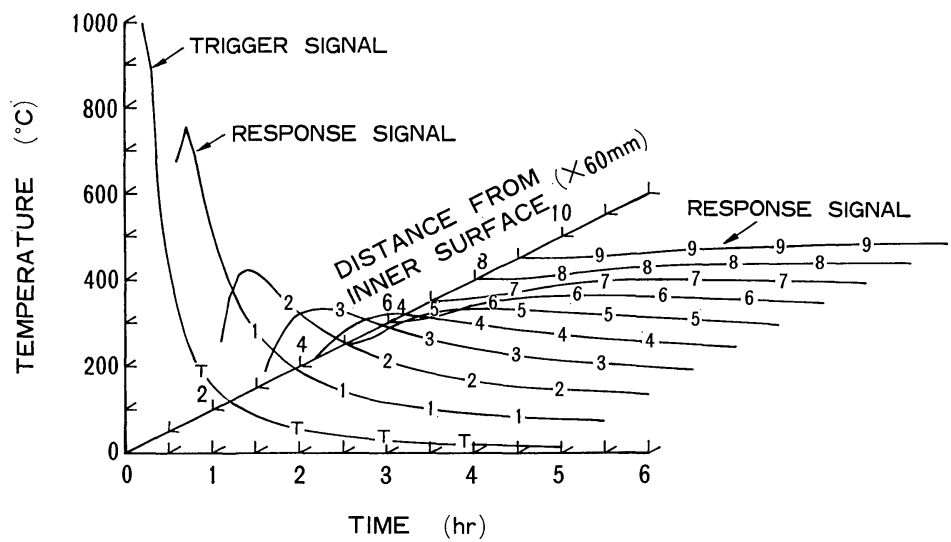

Fig. 2. Heat transfer and temperature profile in the lining.

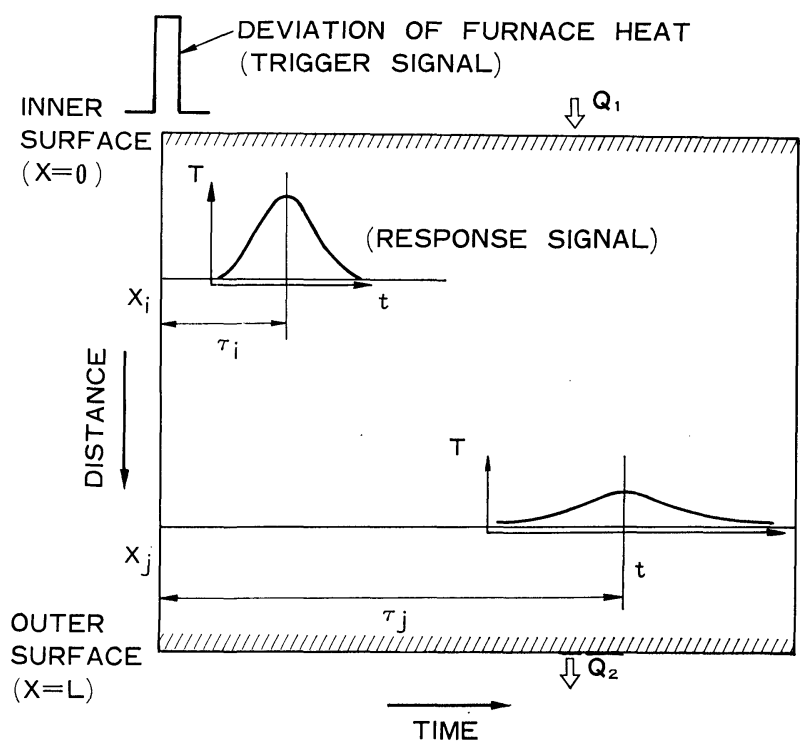

Fig. 4. Relation of time-delay with distance from the inner surface of the lining. each point in the lining after a certain time-delay, $\mathcal{T}_{i}$, which depends on the distance, $T_{i}$, from the inner surface. This phenomenon is shown schematically in Fig. 2.

The approximate temperature profile in the lining is found by solving the following equation for transient heat conduction:

$$
\begin{aligned}
\frac{\partial T}{\partial t} & =\alpha \frac{\partial^{2} T}{\partial X^{2}} \ldots \ldots \ldots \ldots \ldots \ldots \ldots \\
\left.\frac{\partial T}{\partial X}\right|_{X=0} & =\frac{Q_{1}}{h_{1}},\left.\frac{\partial T}{\partial X}\right|_{X=L}=\frac{Q_{2}}{h_{2}}
\end{aligned}
$$

where, $Q_{1}$ : the heat-transfer rate through the inner surface induced by heat variation in a furnace

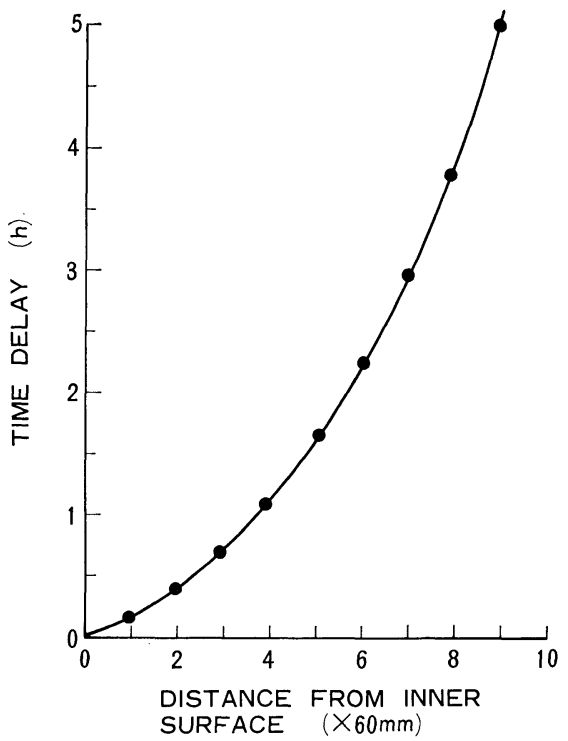

Fig. 3. Variation of temperature at each sensing point in the lining.

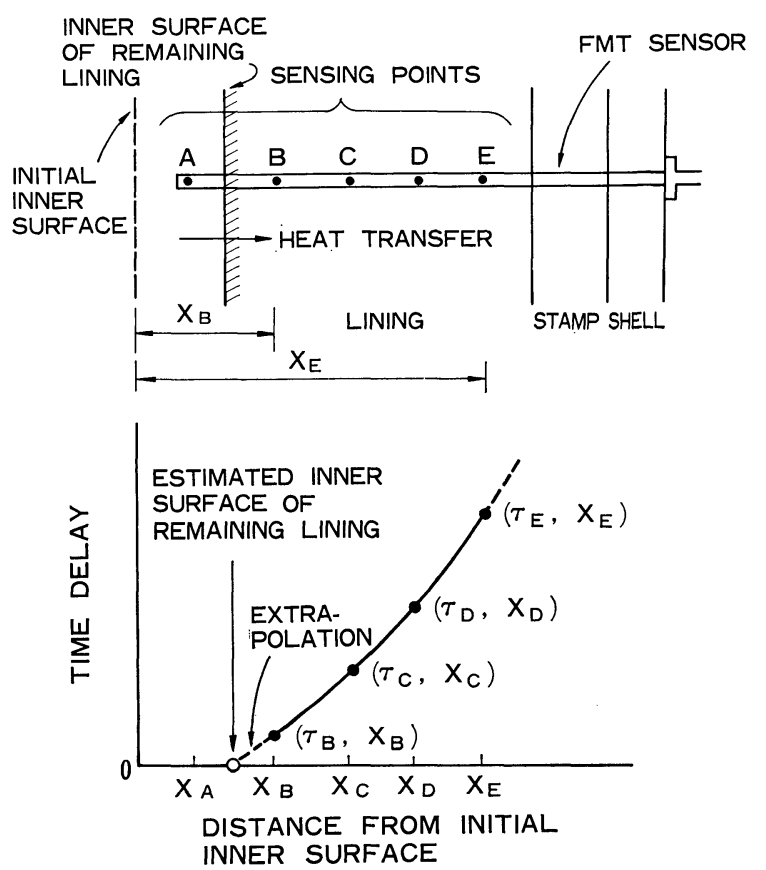

Fig. 5. Principles of the Trigger Response Method. 
$Q_{2}$ : the heat-dissipation rate through the outer surface

$\alpha$ : the thermal diffusivity

$h$ : the heat transfer coefficient at each surface

$L$ : the lining thickness.

The calculated values of the temperature at each sensing point in the lining are illustrated in Fig. 3.

The maximum of temperature in Fig. 3 is plotted in Fig. 4, where the ordinate is the time-delay and the abscissa is the distance from the inner surface. As shown in Fig. 4, the time-delay is zero on the inner surface.

Therefore, if the FMT sensor is installed in the lining (as shown in Fig. 5) and the time-delay, $\tau_{i}$ (between the "Trigger signal ", $\mathcal{T}_{t}$, and the "Response signal ", $T_{r i}$, at each sensing point, $X_{i}$, in the lining), is calculated, we can estimate the position of the inner surface of the remaining lining by extrapolating the $\tau-X$ curve (Fig. 5).

The time-delay $\tau_{i}$ is equal to the extreme point of the cross correlated coefficient between $T_{t}$ and $T_{r i}$. We have named this method the "Trigger Response Method". Figure 6 shows the analytical procedure

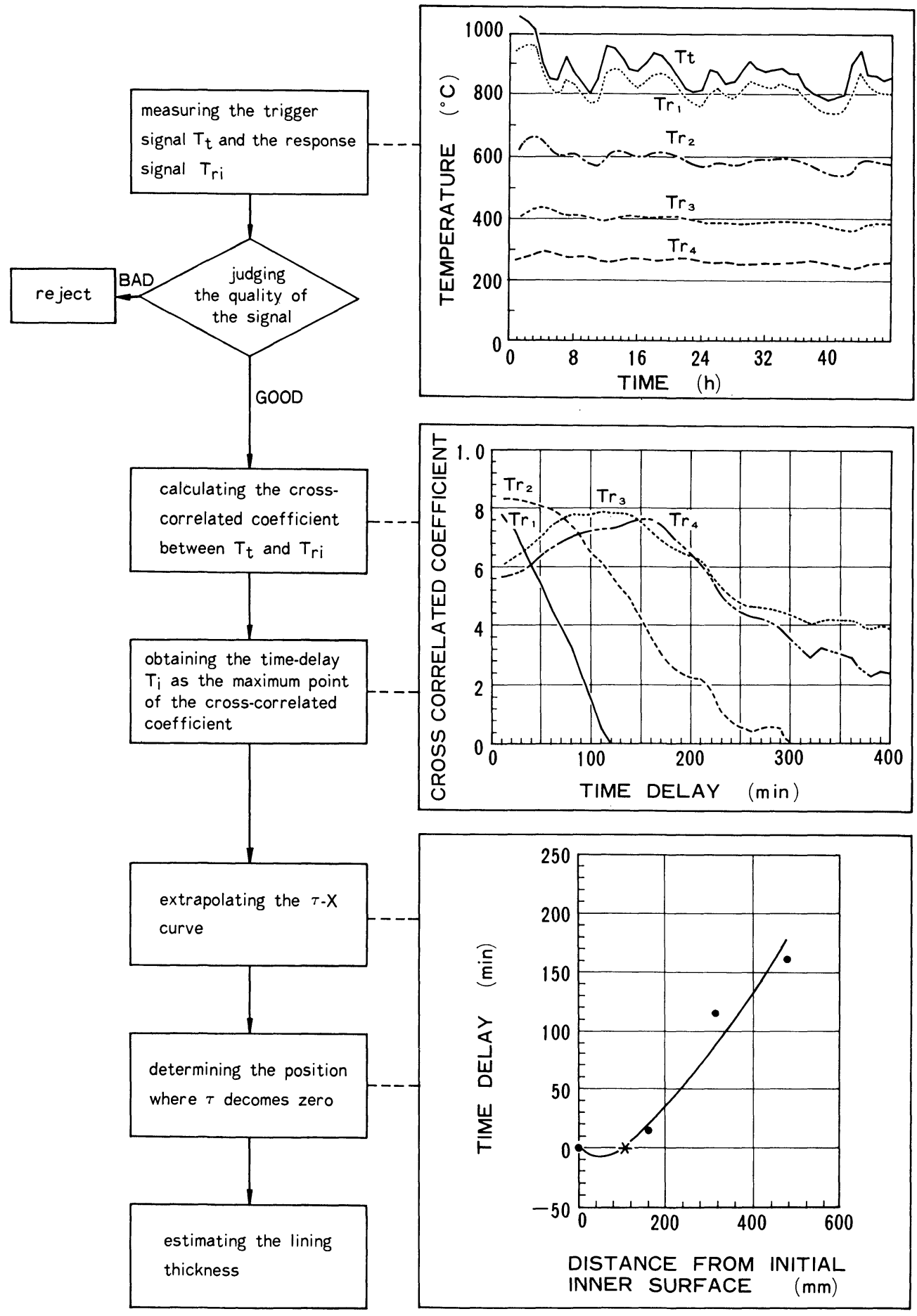

Fig. 6. Analytical procedure for estimating the lining thickness by the Trigger Response Method. 
for estimating the lining thickness by the Trigger Response Method.

As the Trigger Response Method is based only on the cross correlation between temperature signals in the lining, the estimation of the lining thickness is not influenced by the sequential change in the physical properties of the lining materials during the operation of a furnace.

\section{Method of the Field Test and Its Results}

The FMT sensor and The Trigger Response Method were applied during a 19 month field test in the shaft of No. 1 Blast Furnace of Kobe Works (capacity $904 \mathrm{~m}^{3}$; tertiary blowing July, 1977), Fig. 7 shows the arrangement of the sensors which were applied to the shaft of the No. 1 Blast Furnace. Two sets of the FMT sensors were installed at the same height (GL $+17000 \mathrm{~mm}, \mathrm{~S}-\mathrm{W}$ ) and were located $1 \mathrm{~m}$ away from each other on the circumference.

The signals $\left(T_{1}\right)$ from the temperature sensing points that protrude inside the furnace were regarded as the Trigger signals and the signals from the temperature sensing points in the lining were regarded as the Response signals. These signals from the FMT sensors were recorded by a data logger and a chart recorder; the time-delay between the Trigger signals and the Response signals was analyzed by means of a computer; then, the remaining lining thickness was estimated by the Trigger Response Method. Figures 8 and 9 show typical examples of the output from the FMT sensor, and the $\tau-X$ curve which was obtained

KOBE 1 BF SHAFT-L

$(\mathrm{GL}+17,000: \mathrm{S}-\mathrm{W})$

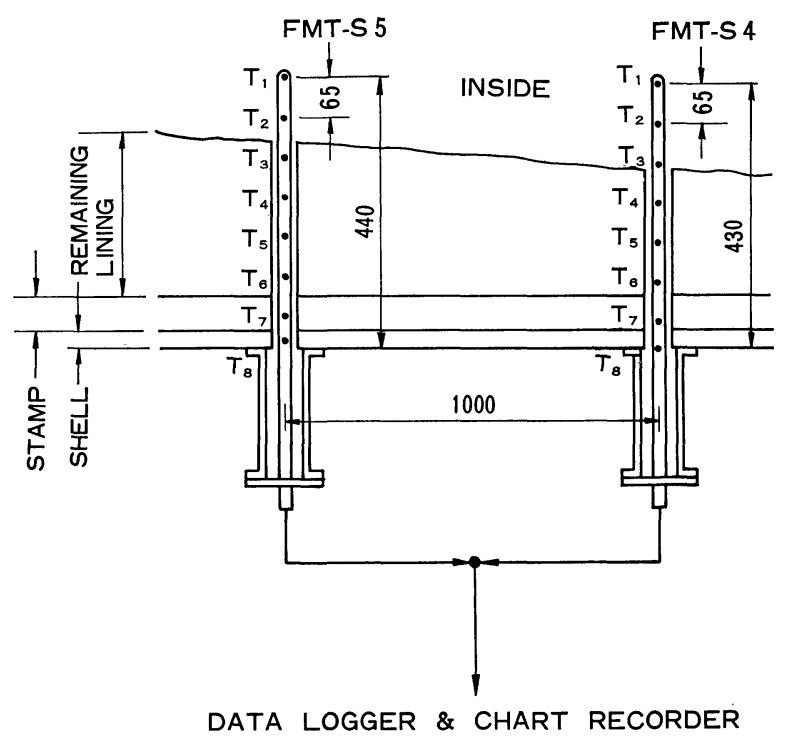

Fig. 7. Installation of the FMT sensor in the lining.
Fig. 8.

Recorded temperature signal from the FMT sensor.

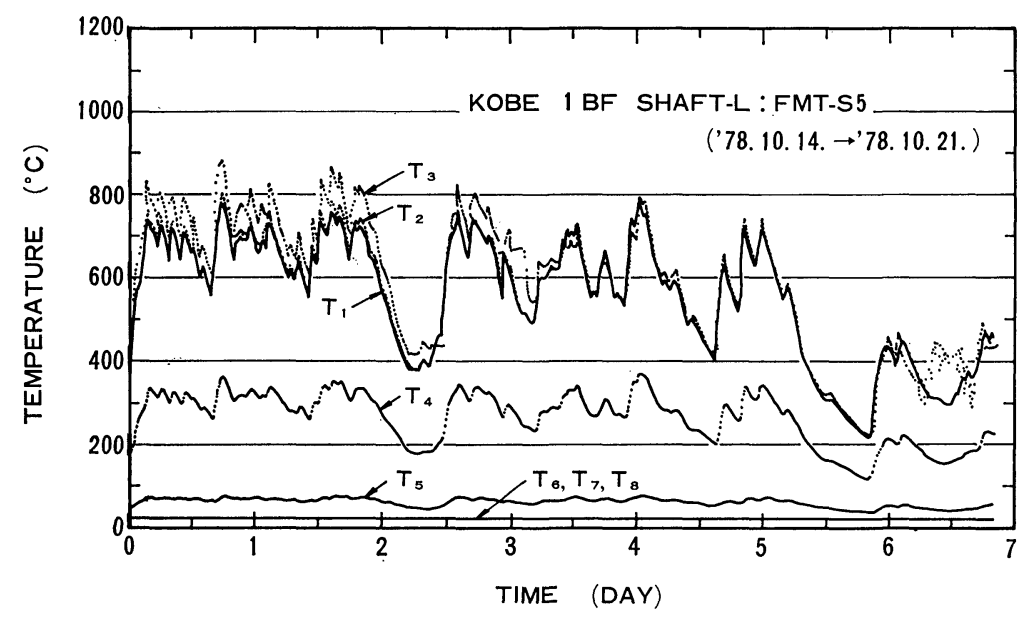

Fig. 9.

Recorded temperature signal from the FMT sensor.

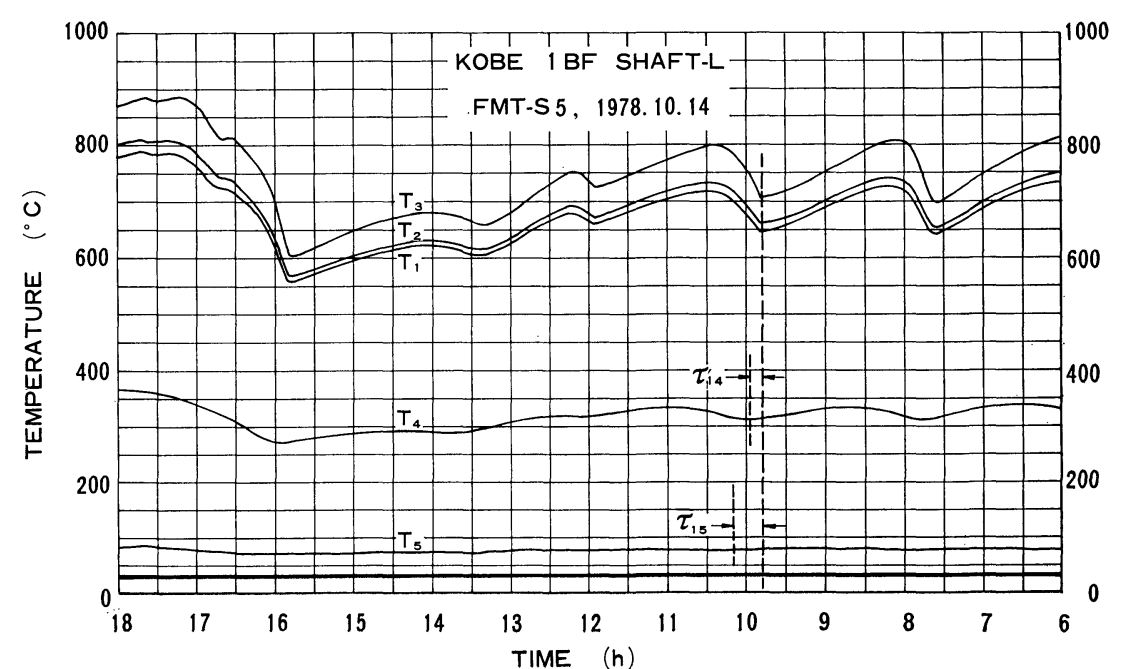


from the data is shown in Fig. 10.

Figure 11 shows the sequential changes in the remaining thickness based upon these results. Figure 11 also shows the remaining lining thickness that was measured by boring at the beginning of the field test (August, 1978) and also at the end of the test (March, 1980). The practicability of the technique was confirmed when the measurements taken by boring coincided with their estimation by the Trigger Response Method, which agreed within an error tolerance of less than $5 \%$.

\section{Measurements of the Profile of Lining Ero- sion at the Hearth}

It is very important to estimate the profile of lining erosion at the hearth of a blast furnace because the erosion of refractory lining can lead to a catastrophic

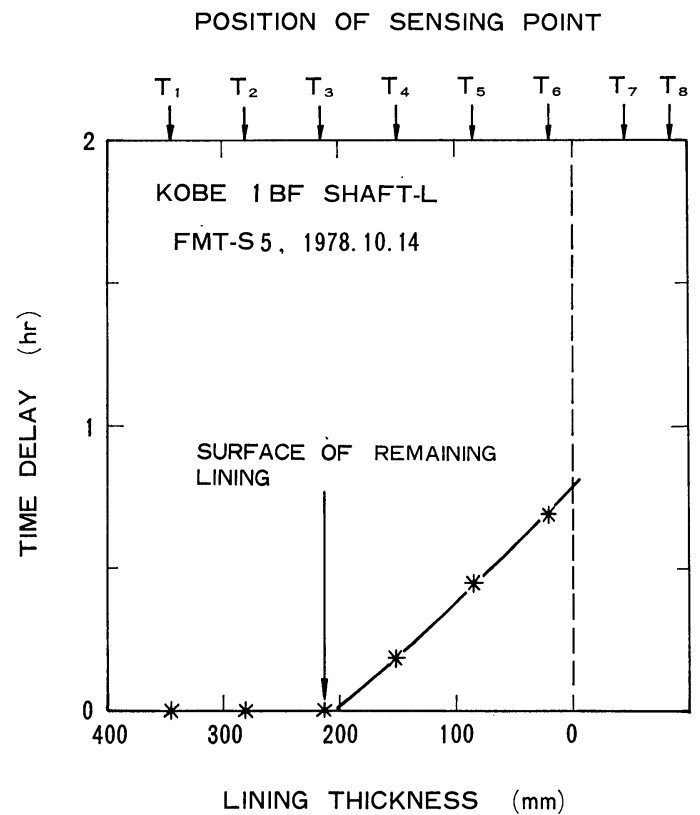

Fig. 10. Relation of time-delay with position of sensing point.

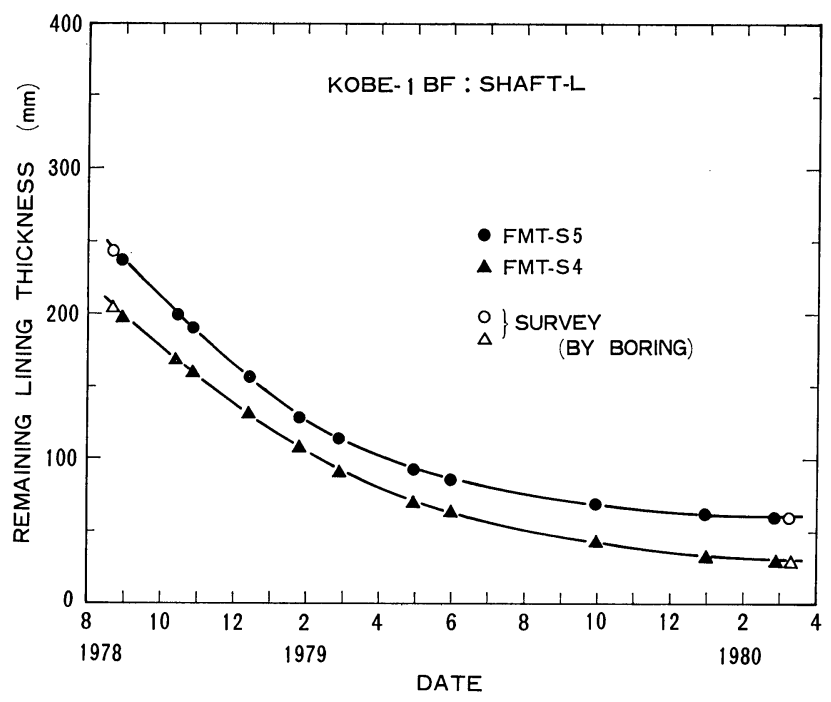

Fig. 11. Sequential changes of remaining lining thickness. accident. Conventionally, the profile of lining erosion at the hearth was estimated for the isothermal line of the solidifying point of iron by the measuring of temperature or heat flow near the shell. The hearth, however, is distinct from the shaft in having corners, making it difficult to apply the one-dimensional heat conduction analysis, that leads to unsatisfactory correspondence with the results of blast furnace dissection studies.

The finite element method is known as a general method for calculating two-dimensional temperature distribution in a solid. Nevertheless application of the finite element method for estimating the profile of lining erosion at the hearth is difficult, since it is premised upon the input data of boundary conditions which themselves are the desired results. Even if the finite element method is applied, the on-line analysis can involve problems of overly large calculation capacity and time. In order to solve these problems, the Profile Coefficient Method has been developed along with on-line analyzing system which can estimate the profile of lining erosion at the hearth.

\section{Principles of Analysis by the Profile Coefficient Method}

The Profile Goefficient Method employs a polar coordinate $\left(R_{i}, \theta_{i}\right)$, which is shown in Fig. 12, to express the profile of lining erosion which is defined by solidifying point $1140^{\circ} \mathrm{C}$ of iron, where the origin $P$ of the polar coordinate is located at the point near the furnace center above the corner of the hearth and $\theta_{i}$ is divided into equal angles.

If the value of erosion point $R_{i}(i=1,2 \ldots, \mathcal{N})$ is directly calculated from the following functional equation,

$$
R_{i}=f_{i}\left(T_{1}, T_{2}, \ldots, T_{M}\right), \quad i=1,2, \ldots, \mathcal{N}
$$

by substituting data of $T_{j}(j=1,2 \ldots, M)$ which is measured by means of thermometers installed in the lining of the hearth, the on-line analysis of the profile of lining erosion can be realized.

By expanding the right side of Eq. (3) in the Tailor series and by cutting off the term of second order and further, the resulting equation is

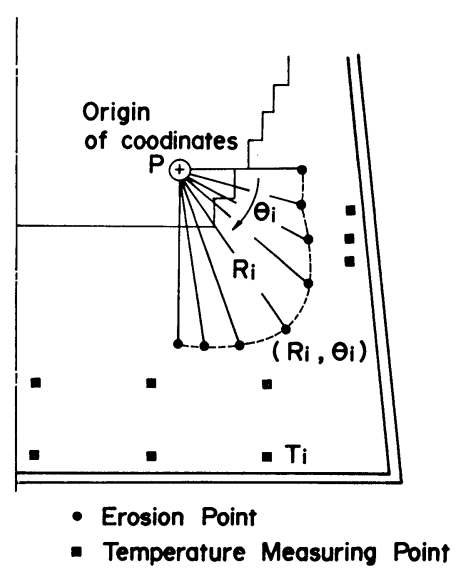

Fig. 12. Representation of the profile of lining erosion on the Profile Coefficient Method. 


$$
\begin{aligned}
R_{i}= & f_{i}\left(T_{10}, \mathcal{T}_{20}, \ldots, \mathcal{T}_{M 0}\right) \\
& +\sum_{j=1}^{M}\left[\left(\frac{\partial f_{i}}{\partial T_{j}}\right)_{T_{j}=T_{j} o} \cdot\left(T_{j}-\mathcal{T}_{j o}\right)\right)
\end{aligned}
$$

where $T_{10}, T_{20}, \ldots, T_{M 0}$ are constants that correspond to measured temperatures at the certain time, $f_{i}\left(T_{10}, T_{20}, \ldots, T_{M 0}\right)$ is given from the solutions of the finite element method with the indirect method in which the above temperatures are used for boundary conditions.

If $f_{i}\left(T_{10}, T_{20}, \ldots, T_{M 0}\right)$ and $\left(\partial f_{i} / \partial T_{j}\right)_{T_{j}=T_{j 0}}$, which have been named the Profile Coefficient, are calculated in advance through the off-line analysis, the profile of lining erosion at the hearth can be estimated by using Eq. (4) when the terms of second order of $\left(\mathcal{T}_{j}-\mathcal{T}_{j o}\right)$ and further are neglected. Furthermore, when the erosion advance over the present distance, namely over the range where the terms of second order of $\left(T_{j}-T_{j o}\right)$ and further can be neglected, the on-line analysis will be performed successfully with the same accuracy by using a renewed table of the profile coefficients which are recalculated on the off-line analysis from the newly measured temperatures.

\section{Application to Blast Furnace}

In shutting down the No. 1 Blast Furnace of Kakogawa Works, the Profile Coefficient Method was applied by utilizing the existing temperature measuring points, and it was compared with the dissection studies. An example of the results is shown in Fig. 13. The profile of lining erosion $\left(1140^{\circ} \mathrm{C}\right.$ isothermal line) resulting from the Profile Coefficient Method and the profile of iron intrusion resulting from the dissec- tion studies show some deviation at step R8. Considering that the existing temperature measuring points were not the most suitable arrangement for analysis with the Profile Goefficient Method, two profile can be said to be in good coincidence.

After the investigation of a better arrangement of

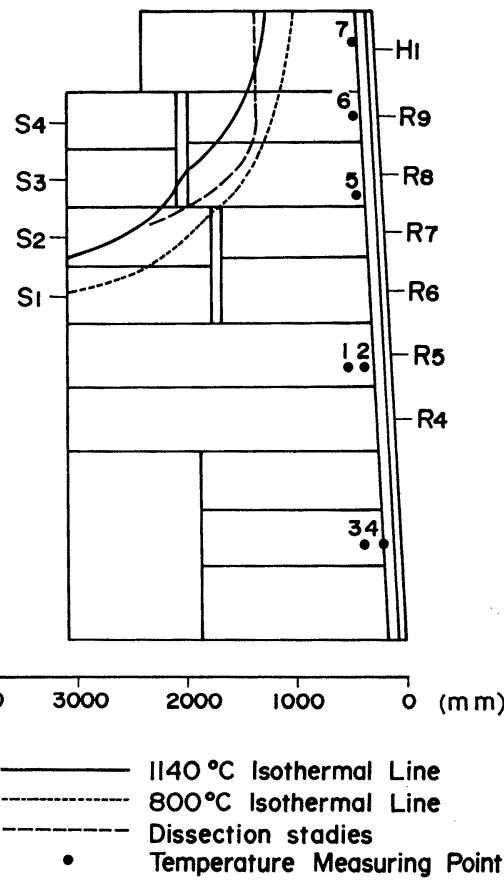

Fig. 13. Analytical result of lining erosion at the hearth of No. 1 Blast Furnace of Kakogawa Works by the Profile Coefficient Method.

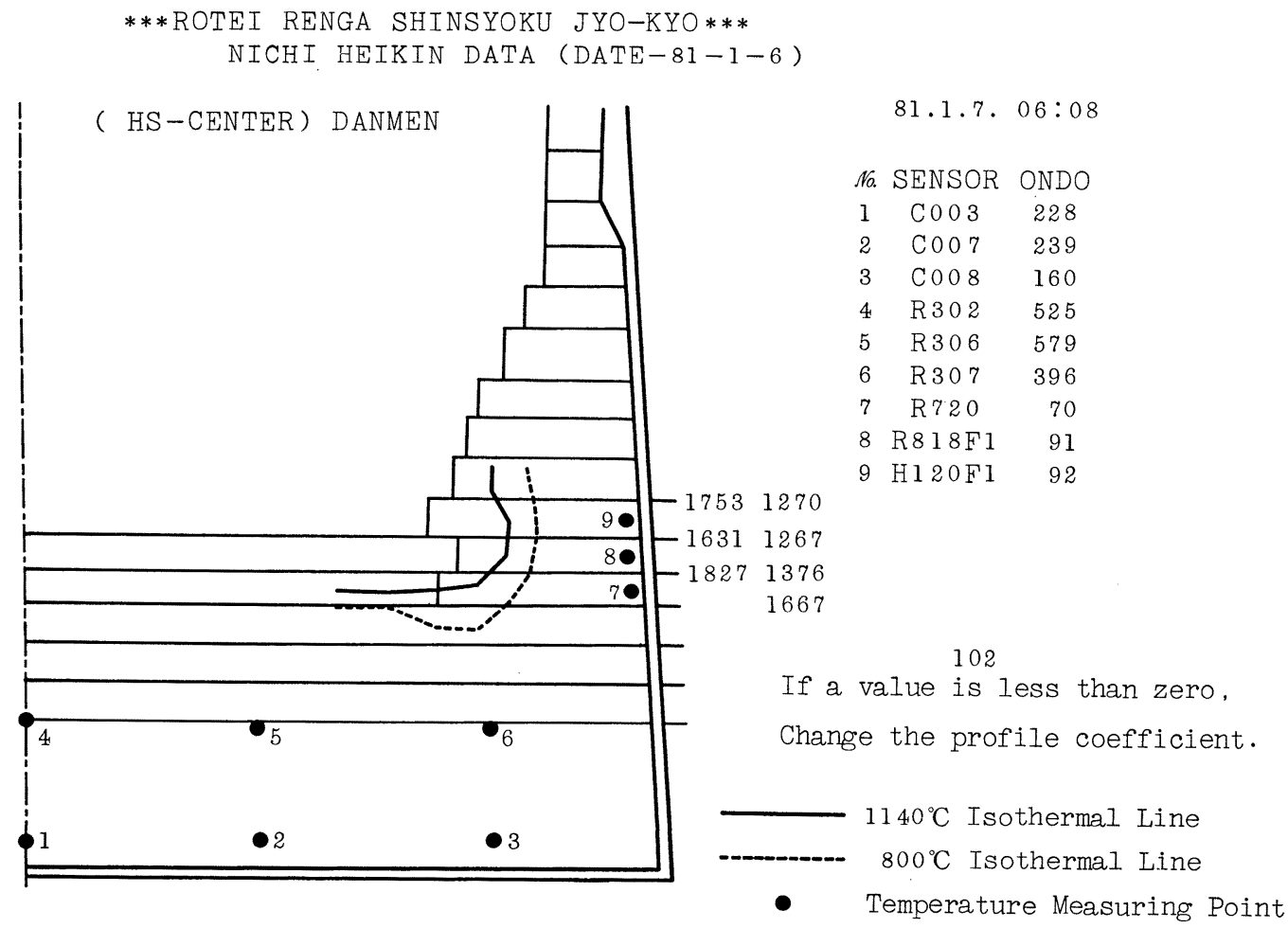

Fig. 14. Analytical result of lining erosion at the hearth of No. 2 Blast Furnace of Kakogawa Works by the Profile Coefficient Method (GRT display). 
temperature measuring points suitable for the analysis, the Profile Coefficient Method has been applied to the No. 2 Blast Furnace of Kakogawa Works. An example of CRT representation is shown in Fig. 14.

\section{Conclusion}

We have developed a sensor (FMT sensor) which can measure the temperature variations simultaneously at a number of positions across the thickness of a blast furnace lining. We also have developed two method of estimating lining erosion by analyzing the signals from the FMT sensor. One of these methods, the Trigger Response Method, can calculate the remaining thickness of refractory lining in the shaft by analyzing the time-delay of temperature variation in the lining. The other method, the Profile Coefficient Method, can estimate the profile of lining erosion at the hearth by introducing the profile coefficient defined by the solution of the finite element method with the indirect method in which measured temperature in the lining are used for boundary conditions.

Based on these techniques, an on line system can monitor the lining erosion at any time has been developed, and its satisfactory performance has been demonstrated in the No. 2 Blast Furnace of Kakogawa Works.

\section{REFERENCES}

1) T. Shibuya: Tetsu-to-Hagané, 67 (1981), A109.

2) Y. Sakamoto, Y. Tamura, T. Sakai, T. Yamamoto and T. Bangi: Tetsu-to-Hagané, 63 (1977), S499.

3) H. Kubo, T. Nishiyama, T. Ikeno, K. Tanaka, M. Yasuno and K. Nakamura: Tetsu-to-Hagané, 65 (1979), S538.

4) J. Ohno: Tetsu-to-Hagané, 58 (1972), 1705. 